\title{
NEURAL NETWORK AUGMENTED IDENTIFICATION OF UNDERWATER VEHICLE MODELS
}

\author{
Pepijn W.J. van de Ven ${ }^{1}$ Tor A. Johansen ${ }^{2}$ \\ Asgeir J. Sørensen ${ }^{3}$ Colin Flanagan $^{1}$ Daniel Toal $^{1}$ \\ ${ }^{1}$ Department of Electronic and Computer Engineering \\ UL, Limerick, Ireland \\ ${ }^{2}$ Department of Engineering Cybernetics \\ NTNU, Trondheim, Norway \\ ${ }^{3}$ Department of Marine Technology \\ NTNU, Trondheim, Norway \\ pepijn.vandeven@ul.ie,tor.arne.johansen@itk.ntnu.no, \\ asgeir.sorensen@marin.ntnu.no,colin.flanagan@ul.ie, \\ daniel.toal@ul.ie
}

\begin{abstract}
In this article the use of neural networks in models for underwater vehicles is discussed. Rather than using a neural network parallel to the known model to account for unmodeled phenomena in a model wide fashion, knowledge regarding the various parts of the model is used to apply neural networks for those parts of the model that are most uncertain. As an example, the damping of an underwater vehicle is identified using neural networks. The performance of the neural network based model is demonstrated for an AUV that changes its physical characteristics during a simulated intervention operation.
\end{abstract}

Keywords:

Autonomous vehicles, Backpropagation, Marine systems, Neural networks, Nonlinear systems, System identification, Time-varying systems

\section{INTRODUCTION}

In recent years highly sophisticated non-linear control schemes for marine vehicles have been developed and implemented. Although modeling of marine vehicles is widely addressed, several parameters still pose uncertainties due to the absence of accurate models. Certain model parameters can be determined analytically. Other parameters, however, will need to be determined using numerical methods or identified using (scaled) model or full scale tests. Both methods can be time consuming and expensive. On top of this, many of the parameters are highly dynamic. Of prime importance in this context is the depen- dence of many hydrodynamic parameters and coefficients on varying velocity regimes, proximity to the sea bed, sea surface and other structures, just to mention a few. At present, models are normally only valid for a limited region of operational conditions. To overcome these problems, neural networks can be used as they offer a means of parameter identification without the necessity of detailed model knowledge. As a result they can identify the parameters of interest over the full region of operation. The application of neural networks for control and modeling (Narendra and Parthasarathy, 1990) has been given considerable attention in recent years. The reader is referred to Van de Ven et al. (2003) and the references 
therein for research endeavours applying neural networks in the field of underwater vehicle control. Rather than using a neural network to approximate unmodeled phenomena in parallel to the total vehicle model, the use of neural networks to model specific parameters in the model, is proposed. Although this approach is not new, see e.g. Psichogios and Ungar (1992) and Thompson and Kramer (1994), little attention is paid to it in the underwater vehicle literature.

\section{ROV KINEMATICS, DYNAMICS AND HYDRODYNAMICS}

In Fossen (2002) and Sørensen and Ronæss (2000) it was shown that the non-linear dynamic equations of motion of a marine vehicle in six degrees of freedom can be expressed in vector notation as:

$$
\mathbf{M} \dot{\boldsymbol{\nu}}+\mathbf{C}(\boldsymbol{\nu}) \boldsymbol{\nu}+\mathbf{D}(\boldsymbol{\nu}) \boldsymbol{\nu}+\mathbf{g}(\boldsymbol{\eta})=\boldsymbol{\tau}
$$

with the kinematic equation

$$
\dot{\boldsymbol{\eta}}=\mathbf{J}(\boldsymbol{\eta}) \boldsymbol{\nu}
$$

relating the linear and angular velocity in the Earth-fixed reference frame and the body-fixed reference frame, where:

$$
\begin{array}{lll}
\boldsymbol{\eta} & = & \text { position and orientation of the } \\
& \text { vehicle in the Earth-fixed frame } \\
\boldsymbol{\nu} & =\begin{array}{l}
\text { linear and angular velocity of the } \\
\text { vehicle in the body-fixed frame }
\end{array} \\
\mathbf{M}= & \text { inertia matrix including added mass } \\
\mathbf{C}(\boldsymbol{\nu})= & \text { matrix consisting of Coriolis and } \\
& \text { centripetal terms } \\
\mathbf{D}(\boldsymbol{\nu})= & \begin{array}{l}
\text { matrix consisting of damping or } \\
\text { drag terms }
\end{array} \\
\mathbf{g}(\boldsymbol{\eta})= & \text { vector of restoring forces and mo- } \\
& \text { ments due to gravity and buoyancy } \\
\boldsymbol{\tau}= & \text { vector of control inputs }
\end{array}
$$

In (1) it is assumed that no water current is present. Introducing the latter with velocity $\boldsymbol{\nu}_{c}$ results in the added mass contribution to the Coriolis matrix and the damping matrix to be a function of the relative velocity defined as:

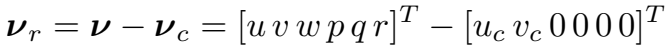

For brevity, and without loss of generality, in this article the current velocity is assumed to be zero. A detailed derivation of the non-linear equations of motion can be found in Fossen (2002). Below a small summary of the model is given.

In the matrix $\mathbf{M}$ two inertial components are accounted for,

$$
\mathbf{M}=\mathbf{M}_{R B}+\mathbf{M}_{A} .
$$

The rigid body inertial matrix, $\mathbf{M}_{R B}$, represents the mass and inertia terms. Added mass is accounted for by the matrix $\mathbf{M}_{A}$.

For the matrix $\mathbf{C}(\boldsymbol{\nu})$, a similar discourse can be held. Both the Coriolis and the centripetal forces are functions of the rigid body mass and added mass and the velocity, $\boldsymbol{\nu}$.

$$
\mathbf{C}(\boldsymbol{\nu})=\mathbf{C}_{R B}(\boldsymbol{\nu})+\mathbf{C}_{A}(\boldsymbol{\nu}) .
$$

$\mathbf{C}_{R B}(\boldsymbol{\nu})$ accounts for rigid body while $\mathbf{C}_{A}(\boldsymbol{\nu})$ accounts for the added mass.

In the damping matrix, $\mathbf{D}(\boldsymbol{\nu})$, four terms are combined:

$$
\mathbf{D}(\boldsymbol{\nu})=\mathbf{D}_{P}+\mathbf{D}_{S}(\boldsymbol{\nu})+\mathbf{D}_{W}+\mathbf{D}_{M}(\boldsymbol{\nu}),
$$

where:

$$
\begin{aligned}
& \mathbf{D}_{P}=\text { potential damping } \\
& \mathbf{D}_{S}(\boldsymbol{\nu})=\text { linear and quadratic skin friction } \\
& \mathbf{D}_{W}=\text { wave drift damping } \\
& \mathbf{D}_{M}(\boldsymbol{\nu})=\text { damping due to vortex shedding }
\end{aligned}
$$
Accurate calculation of these phenomena is difficult. Hence, often the damping is approximated by a diagonal matrix containing the linear and quadratic damping terms according to:

$$
\begin{aligned}
\mathbf{D}(\boldsymbol{\nu})= & -\operatorname{diag}\left\{X_{u}, Y_{v}, Z_{w}, K_{p}, M_{q}, N_{r}\right\} \\
& -\operatorname{diag}\left\{X_{u|u|}|u|, Y_{v|v|}|v|, Z_{w|w|}|w|,\right. \\
& \left.K_{p|p|}|p|, M_{q|q|}|q|, N_{r|r|}|r|\right\} .
\end{aligned}
$$

Although (7) is a good approximation for decoupled motion, for manoeuvres involving movements along and about several body axes at a time, such simple models might prove to be insufficient.

\section{IDENTIFICATION OF MODEL PARAMETERS}

As mentioned, both $\mathbf{M}_{R B}$ and $\mathbf{C}_{R B}(\boldsymbol{\nu})$ can be calculated if the physical characteristics of the vehicle are known. However, proper determination of $\mathbf{M}_{A}$ (from which $\mathbf{C}_{A}(\boldsymbol{\nu})$ can be calculated) requires either the use of dedicated hydrodynamic software (Faltinsen, 1990), or using model or full scale tests to identify the added mass coefficients. In numerical calculations, the vehicle may be divided up into small sections and two dimensional added mass contributions are calculated for those sections. Consecutively, an integration over the whole body yields the three dimensional added mass parameters. In order to apply this method, which is called strip theory, the user is required to provide a detailed description of the vehicle in the form of a CAD drawing. On top of this, slender body theory should be assumed. This part of the modeling process alone can take up considerable time. For bluff bodies, however, other methods must be used. Measuring the added mass parameters using e.g. free decay tests is another option. Up to date there are, to the authors' knowledge, no methods available to perform those tests for all coupled six degrees of freedom simultaneously. On top of the above mentioned problems it should be kept in mind that no means of online updating 
of parameters is available from either method. This possibly even effects the analytically derived values for $\mathbf{M}_{R B}$ and $\mathbf{C}_{R B}(\boldsymbol{\nu})$ as the physical characteristics may change from mission to mission, or during one and the same mission, due to changing payloads. As an illustration to this, one might think of an ROV or an AUV sent on a salvage mission with the aim of lifting an object from the sea floor. Due to lifting the object, both mass and geometrical characteristics of the vehicle will change, thus changing $\mathbf{M}_{R B}$ and $\mathbf{C}_{R B}(\boldsymbol{\nu})$.

\section{SYSTEM IDENTIFICATION USING NEURAL NETS}

Neural networks can be applied both as control plant models and as controllers. Normally the neural network is used parallel to conventional models or controllers in a switching or outputblending fashion. In both cases, however, the neural networks make no, or only partial, use of the available a priori knowledge. Due to looking at the neural network as some non-linear mapping between the plant's input data and output data, knowledge regarding the dependence between parameters is lost. This might lead to an unnecessarily complicated function to be learned by the neural network. As briefly discussed in section 2 several (matrix) parameters related to the rigidbody dynamic equations of motion can be calculated accurately. It is thus intuitively appealing to use a neural network in parallel with a model of the known part of the system dynamics. To illustrate the effect of a neural network placed in parallel with the system model, the process dynamics are expressed in state-space form. To obtain a state space representation of the vehicle,

(1) can be written in the following form:

$$
\dot{\boldsymbol{\nu}}=\mathbf{M}^{-1}[\boldsymbol{\tau}-\mathbf{C}(\boldsymbol{\nu}) \boldsymbol{\nu}-\mathbf{D}(\boldsymbol{\nu}) \boldsymbol{\nu}-\mathbf{g}(\boldsymbol{\eta})] .
$$

Taking the state vector to be $\boldsymbol{\nu}$ and the inputs to the system as: $\boldsymbol{\tau}(t)$ and $\boldsymbol{\eta}(t),(8)$ can be written in state-space form:

$$
\begin{aligned}
& \dot{\boldsymbol{\nu}}=\boldsymbol{\Phi}[\boldsymbol{\nu}(t), \tau(t), \eta(t)] \\
& \mathbf{y}=\boldsymbol{\Psi}[\boldsymbol{\nu}(t)],
\end{aligned}
$$

with $\boldsymbol{\Phi}[\boldsymbol{\nu}(t), \tau(t), \eta(t)]$ the right hand side of (8) and $\boldsymbol{\Psi}[\boldsymbol{\nu}(t)]$ simply $\boldsymbol{\nu}(t)$. Figure 1 shows the corresponding block diagram. Assuming that one has partial knowledge regarding the function $\mathbf{\Phi}$, a neural network can be used to model the unknown part of the system in parallel to the known part of the system as depicted in figure 2. In this approach one assumes: $\boldsymbol{\Phi}=\boldsymbol{\Phi}_{M}+\hat{\boldsymbol{\Phi}}$ where $\boldsymbol{\Phi}_{M}$ corresponds to the known part of $\boldsymbol{\Phi}$ and $\hat{\boldsymbol{\Phi}}$ to the unknown part approximated by the neural network. If the same assumption is made for the matrices $\mathbf{M}, \mathbf{C}(\boldsymbol{\nu})$, $\mathbf{D}(\boldsymbol{\nu})$ and $\mathbf{g}(\boldsymbol{\eta}),(8)$ can be written as:

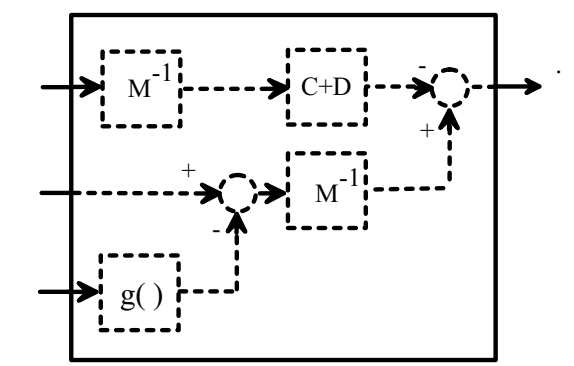

Fig. 1. State space representation of the non-linear dynamic equations of motion

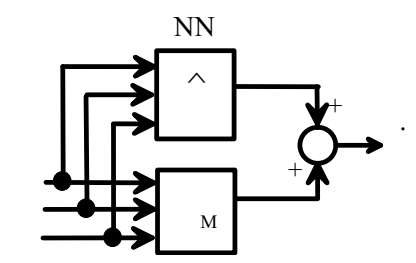

Fig. 2. State space representation of the non-linear dynamic equations with neural network in parallel to model unknown parameters

$$
\begin{aligned}
\dot{\boldsymbol{\nu}} & =\mathbf{M}_{M}^{-1}\left\{\boldsymbol{\tau}-\mathbf{C}_{M}(\boldsymbol{\nu}) \boldsymbol{\nu}-\mathbf{D}_{M}(\boldsymbol{\nu}) \boldsymbol{\nu}-\mathbf{g}_{M}(\boldsymbol{\eta})\right\} \\
& +\mathbf{M}_{M}^{-1}\{-\hat{\mathbf{C}}(\boldsymbol{\nu}) \boldsymbol{\nu}-\hat{\mathbf{D}}(\boldsymbol{\nu}) \boldsymbol{\nu}-\hat{\mathbf{g}}(\boldsymbol{\eta})\} \\
& +\hat{\mathbf{M}}^{-1}\left\{\boldsymbol{\tau}-\mathbf{C}_{M}(\boldsymbol{\nu}) \boldsymbol{\nu}-\hat{\mathbf{C}}(\boldsymbol{\nu}) \boldsymbol{\nu}-\right. \\
& \left.-\mathbf{D}_{M}(\boldsymbol{\nu}) \boldsymbol{\nu}-\hat{\mathbf{D}}(\boldsymbol{\nu}) \boldsymbol{\nu}-\mathbf{g}_{M}(\boldsymbol{\eta})-\hat{\mathbf{g}}(\boldsymbol{\eta})\right\} .
\end{aligned}
$$

The last three lines of (10) represent $\hat{\boldsymbol{\Phi}}$. These terms will be estimated by the neural network. Comparing this approach to the one depicted in figure 3 demonstrates that the required non-linear mapping is unnecessarily complicated. The neural networks in figure 3 namely model:

$$
N N_{1}=\hat{\mathbf{M}}^{-1} ; N N_{2}=\hat{\mathbf{C}}+\hat{\mathbf{D}} ; N N_{3}=\hat{\mathbf{g}}(\eta),
$$

which is a considerably easier task. Another advantage is that use can be made of known parameter features during the learning stage. Hence, the alternative configuration of the neural networks, as shown in figure 3 might prove worthwhile and is therefore used in this study.

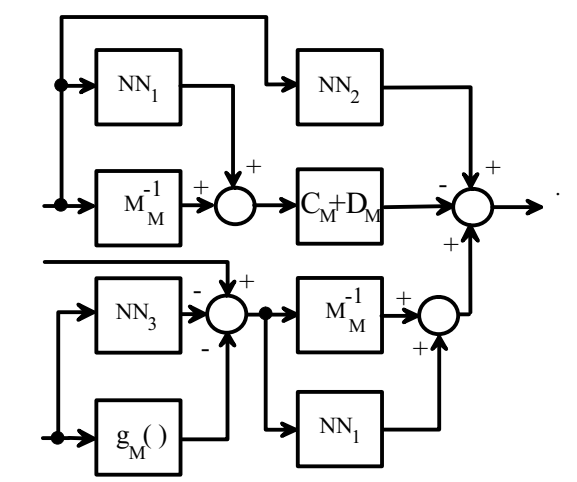

Fig. 3. State space representation of the nonlinear dynamic equations with several neural networks to model unknown parameters 


\section{CASE STUDIES}

In this section the viability of the above outlined method will be tested using various simulation setups. In all simulations, first offline learning was performed to initialise the neural networks. Afterwards, if improvements are expected, online training is performed. The neural networks have 12 input neurons, 5 neurons in one hidden layer and one linear output neuron. The other neurons use a hyperbolic tangent activation function. Both the hidden and the output layer use bias parameters. In the simulations the following assumptions are made:

- Acceleration and velocity of the vehicle can be measured.

- Only the damping matrix is unknown.

- The vehicle can be actuated in all degrees of freedom.

- Open loop simulations are performed.

The parameter that will be approximated by the neural network is the damping matrix. A first advantage of using a neural network is that strong assumptions, as discussed in Section 2 are not made. Higher order terms and coupling between various degrees of freedom can be taken into account by the neural network. To demonstrate this the damping matrix inhibits significant offdiagonal elements and non-linearities. In the first computer experiment, Section 5.1, the damping matrix will be approximated offline using velocity and acceleration data assuming zero measurement noise. Then, in Section 5.2, noise is added to the setup. Online learning will be used to decrease the state prediction error. Finally, in Section 5.3, the beneficial influence of online learning on changing vehicle dynamics will be demonstrated.

Assuming that only the damping matrix $\mathbf{D}(\boldsymbol{\nu})$ is unknown and hence, initially, not accounted for, the dynamic equation for the reference system or process plant model (ppm) and the initial approximate or control plant model $(\mathrm{cpm})$ respectively can be written as:

$$
\begin{aligned}
\dot{\boldsymbol{\nu}}_{p p m}= & \mathbf{M}^{-1}\left[\boldsymbol{\tau}-\mathbf{C}\left(\boldsymbol{\nu}_{p p m}\right) \boldsymbol{\nu}_{p p m}-\right. \\
& \left.\mathbf{D}\left(\boldsymbol{\nu}_{p p m}\right) \boldsymbol{\nu}_{p p m}-\mathbf{g}\left(\boldsymbol{\eta}_{p p m}\right)\right], \\
\dot{\boldsymbol{\nu}}_{c p m}= & \mathbf{M}^{-1}\left[\boldsymbol{\tau}-\mathbf{C}\left(\boldsymbol{\nu}_{c p m}\right) \boldsymbol{\nu}_{c p m}-\mathbf{g}\left(\boldsymbol{\eta}_{c p m}\right)\right],
\end{aligned}
$$

To obtain information regarding the damping matrix, data is generated through simulation of the ppm model with a given input, and onestep-ahead predictions are computed from the cpm model. At every time step one assumes both systems have the same state vector: $\boldsymbol{\nu}_{p p m}=$ $\boldsymbol{\nu}_{c p m}=\boldsymbol{\nu}$. Substituting $\boldsymbol{\nu}$ for $\boldsymbol{\nu}_{p p m}$ and $\boldsymbol{\nu}_{c p m}$ in equations 12 and 13, the difference between equations 12 and 13 becomes the damping matrix
$\boldsymbol{D}(\boldsymbol{\nu})$, multiplied by the state vector $\boldsymbol{\nu}$ and the inverse of the mass matrix, $\boldsymbol{M}^{-1}$. The product $\boldsymbol{D}(\boldsymbol{\nu}) \boldsymbol{\nu}$ can thus be calculated as:

$$
\mathbf{D}(\boldsymbol{\nu}) \boldsymbol{\nu}=\mathbf{M}\left[\dot{\boldsymbol{\nu}}_{c p m}-\dot{\boldsymbol{\nu}}_{p p m}\right]
$$

Equation (14) will be used for training of the neural networks and hence is the identification model.

\subsection{Case Study I: Drag estimation using noise free signals}

To gather training data, a simulation of a trajectory using the process plant model is performed. The vehicle is actuated with $\boldsymbol{\tau}=$ [100 100100101010$]^{T}$ for 15 seconds without performing control. After 15 seconds $\tau$ is set to $\left[\begin{array}{llllll}0 & 0 & 0 & 0 & 0 & 0\end{array}\right]^{T}$. As the vehicle used in this simulation is neutrally buoyant, its velocity gradually returns to $\boldsymbol{\nu}=\left[\begin{array}{lllll}0 & 0 & 0 & 0 & 0\end{array}\right]^{T}$. Both velocity, i.e. the state of the system, and acceleration are recorded and consecutively used to train a neural network offline. As it is known that damping is a function of $\nu|\nu|$, the neural network has both $\nu$ and $|\nu|$ as inputs. The magnitudes of the velocities in the six degrees of freedom are not necessarily of the same order, which possibly leads to complications in the training algorithm. In neural networks trained with back propagation, neurons with smaller training signals tend to be dominated by neurons with larger training signals. As a result the former tend to learn slower and for those neurons the modeling error might thus not decrease properly. To prevent this, six separate networks were used for training. After identification of $\mathbf{D}(\boldsymbol{\nu}) \boldsymbol{\nu}$ in (14) has been performed, the neural networks can be used in the model as shown in figure 4 . The control plant model now becomes:

$$
\dot{\boldsymbol{\nu}}_{c p m}=\mathbf{M}^{-1}[\boldsymbol{\tau}-\mathbf{C}(\boldsymbol{\nu}) \boldsymbol{\nu}-\mathbf{g}(\boldsymbol{\eta})-\hat{\mathbf{D}}(\boldsymbol{\nu}) \boldsymbol{\nu}] .
$$

where $\hat{\mathbf{D}}(\boldsymbol{\nu}) \boldsymbol{\nu}$ indicates that this is the neural network approximation of $\mathbf{D}(\boldsymbol{\nu}) \boldsymbol{\nu}$.

It should be noted that, unlike the other blocks, the block with caption $\hat{\boldsymbol{D}}(\boldsymbol{\nu}) \boldsymbol{\nu}$ should not be interpreted as a multiplication of the inputs and the block's argument. Figure 5 shows open loop predictions made by the vehicle model using a neural network to model damping and the corresponding

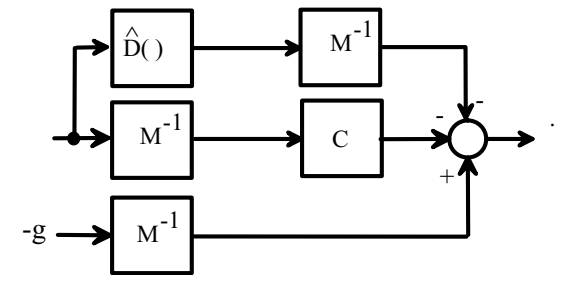

Fig. 4. Model of vehicle with the damping effects modeled by a neural network 

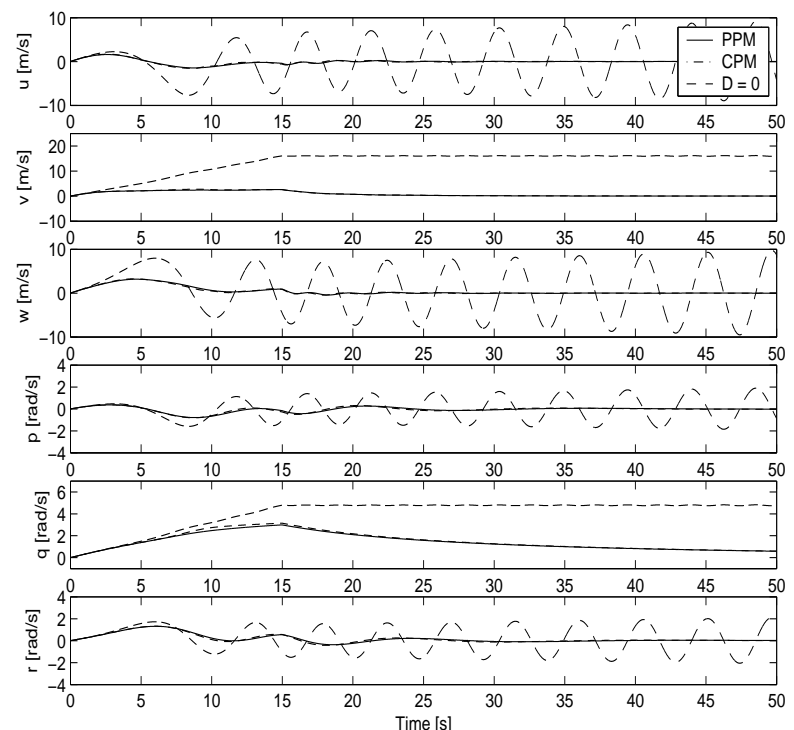

Fig. 5. Prediction of vehicle model using a NN for modeling of the damping

real trajectory. The graphs for the trajectory of the process plant model and the control plant model with neural network are lying on top of each other. It can thus be concluded that the neural networks are capable of approximating damping with sufficient accuracy for extended periods of time. To demonstrate the influence of damping on the vehicle model, figure 5 also shows the model output if the damping is simply taken equal to zero. This graph shows that, as expected, neglecting the damping leads to considerable errors in the state prediction.

\subsection{Case Study II: Drag estimation under noisy conditions}

To investigate the ability of the neural network to perform estimation of the damping using noisy training signals, typical noise is added to the measurements. For the linear accelerations a white noise sequence with an amplitude of $0.5 \mathrm{~ms}^{-2}$ is added. The angular accelerations are summed with a noise component of $0.05 \mathrm{rads}^{-2}$. The velocity measurements are assumed to be impeded with a white noise sequence, which amplitude is equal to $0.1 \%$ of the magnitude of the velocity. It should be noted that in the simulations no noise prefiltering is assumed. This would make matters considerably easier for the neural network. Performance of the model is shown in figure 6. Clearly, the added noise affects the prediction abilities of the model. The prediction of the state can be improved by continuing the learning process online. The result of this experiment is shown in figure 7 , demonstrating that online learning improves the state prediction considerably. However, in this case the neural network does not necessarily represent the damping any more. Online
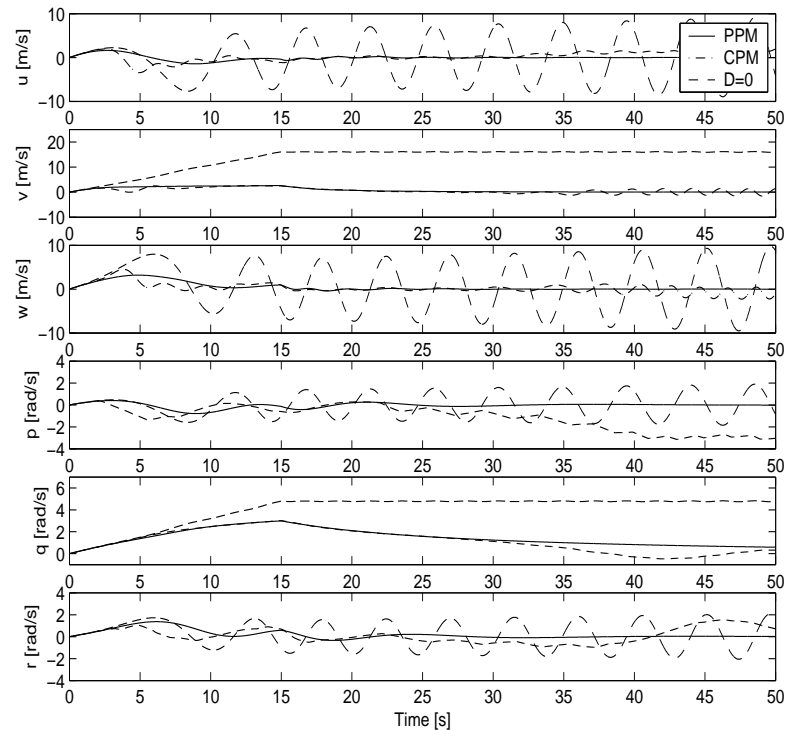

Fig. 6. Prediction of vehicle model using a NN for modeling of the damping under noisy conditions

learning results in a better local estimate of the damping as the neural network is now trained with larger weight on the latest data points. The global estimation accuracy may suffer from this.

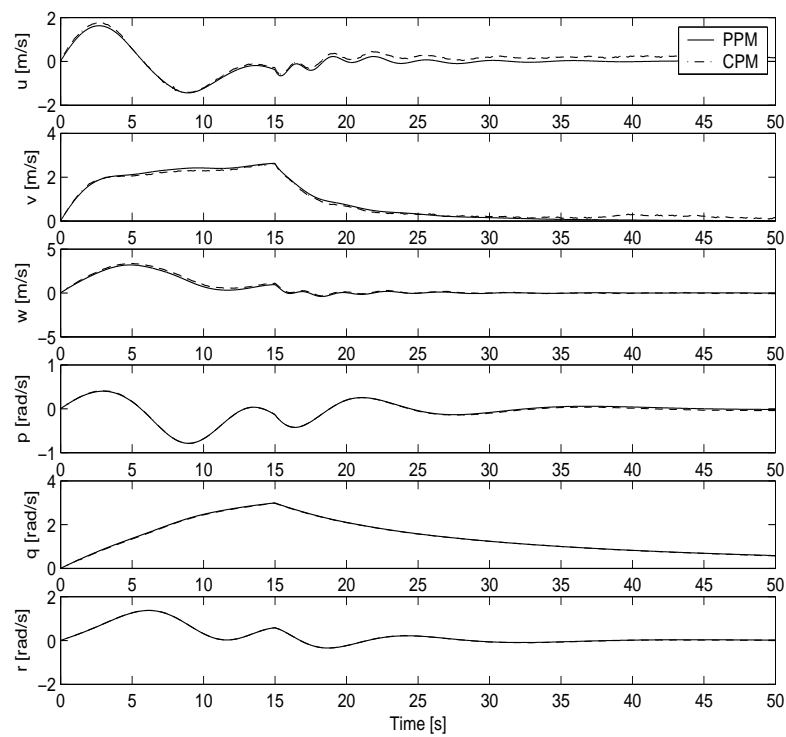

Fig. 7. Prediction of vehicle model using a NN for modeling of the damping under noisy conditions with online learning

\subsection{Case Study III: Drag estimation for time-varying dynamics}

In this simulation an AUV with an offline trained neural network representing damping is sent on a mission. The mission is to pick up a cylinder, with a mass of $60 \mathrm{~kg}$ (while the vehicle mass is $95 \mathrm{~kg}$ ) and transport it to its destination. This process starts at $t=5 \mathrm{~s}$ and results in several matrices to drastically be changed: changes in 


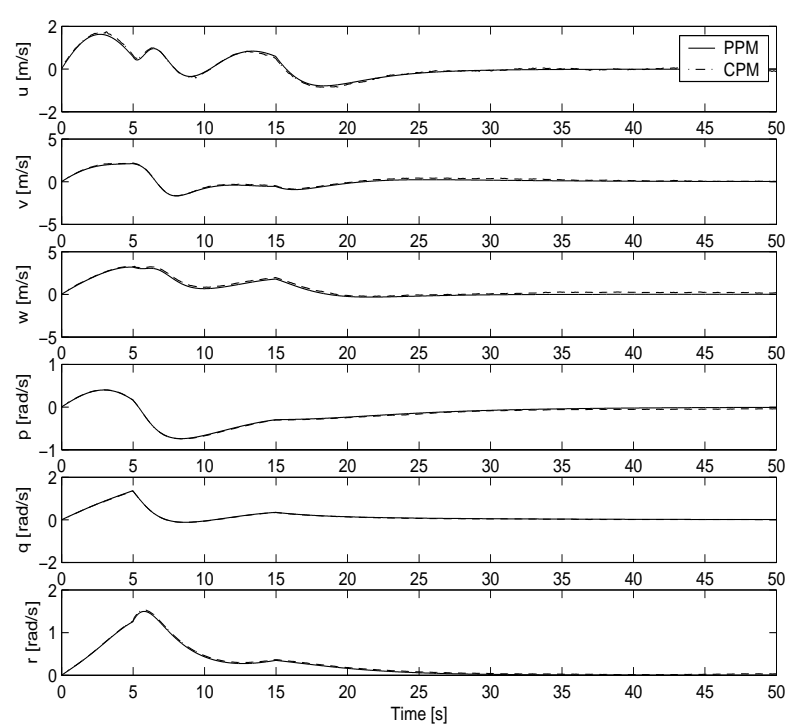

Fig. 8. Prediction of vehicle model using a NN for changing damping coefficients and using online learning

both the mass matrix, $\mathbf{M}$, and the Coriolis matrix, $\mathbf{C}(\boldsymbol{\nu})$, are assumed to be known. Changes in the damping matrix will be assumed to be unknown. Online learning is performed to adapt for the changing damping. Figure 8 shows the trajectories for the process plant model and the control plant model using the neural network to model damping. Although the change in damping is considerable with the various damping parameters changing between a factor 1.5 and 10, only small deviations from the ppm trajectory can be seen. The changes from the trajectory that would have occurred if no online updating of the neural network was performed, are shown in figure 9 . Comparing these two figures demonstrates the benefit of online learning.

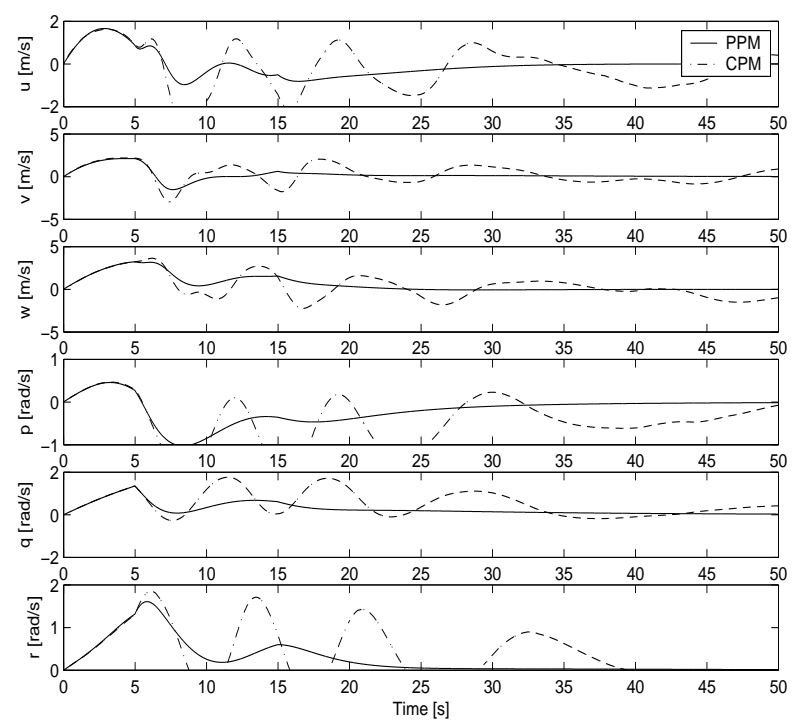

Fig. 9. Prediction of vehicle model using a NN without online learning while damping coefficients change

\section{CONCLUSIONS}

In this work, modeling of the damping of an underwater vehicle was performed using neural networks. Rather than using a neural network in parallel to the whole model (in an attempt to account for all unknown parameters in one neural network), neural networks are used to identify parts of the model that are known to be most uncertain. Under noisy conditions a neural network is trained to approximate the damping matrix. The results show that these neural networks can be used to improve the identification of poorly modeled phenomena. Online learning can be applied to alleviate the influence of noise and to adapt for changing parameters due to e.g. mission objectives. In this study, matters were simplified by assuming other parts of the model to be fully known. Future work will focuss on identification of several uncertainties at a time.

\section{ACKNOWLEDGEMENT}

This work was supported by the EC Research Directorates through a project at the Marie Curie Training Site, CyberMar at NTNU (HPMT-CT2001-00382) and by the Irish Research Council for Science, Engineering and Technology: funded by the National Development Plan.

\section{REFERENCES}

Faltinsen, O.M. (1990). Sea Loads on Ships and Offshore Structures. Cambridge University Press.

Fossen, T.I. (2002). Marine Control Systems. Guidance, Navigation and Control of Ships, Rigs and Underwater Vehicles. 1st ed.. Marine Cybernetics, AS.

Narendra, K.S. and K. Parthasarathy (1990). Identification and control of dynamical systems using neural networks. IEEE Transactions on Neural Networks 1, 4-27.

Psichogios, D. C. and L. H. Ungar (1992). A hybrid neural network - first principles approach to process modeling. AIChE J. 38, 14991511.

Sørensen, A. J. and M. Ronæss (2000). The Ocean Engineering Handbook. Chap. Mathematical modeling of dynamically positioned and thruster assisted anchored marine vessels, pp. 176-89. CRC Press.

Thompson, M. L. and M. A. Kramer (1994). Modeling chemical processes using prior knowledge and neural networks. AIChE J. 40, 1328-40.

Van de Ven, P., C. Flanagan and D. Toal (2003). Artificial intelligence for the control of underwater vehicles. In: Smart Engineering System Design, Proc. of the Artificial Neural Networks in Engineering Conf. pp. 559-64. 